ITF-2002/41

SPIN-2002/23

hep-th/0208054

\title{
ON PECULIARITIES AND PIT FALLS IN PATH INTEGRALS ${ }^{\dagger}$
}

\author{
Gerard 't Hooft \\ Institute for Theoretical Physics \\ Utrecht University, Leuvenlaan 4 \\ 3584 CC Utrecht, the Netherlands \\ and \\ Spinoza Institute \\ Postbox 80.195 \\ 3508 TD Utrecht, the Netherlands \\ e-mail: g.thooft@phys.uu.nl \\ internet: http://wWw.phys.uu.nl/ thooft/
}

\begin{abstract}
Path integrals can be rigorously defined only in low dimensional systems where the small distance limit can be taken. Particularly non-trivial models in more than four dimensions can only be handled with considerable amount of speculation. In this lecture we try to put these various aspects in perspective.
\end{abstract}

November 6, 2018

\footnotetext{
${ }^{1}$ Opening Lecture given at the 7th International Conference on Path Integrals, "From Quarks to Galaxies", Antwerp, 27 to 31 May 2002.
} 


\section{Introduction.}

Although the words "Path Integral" and "Functional Integral" are usually treated as though they were synonyms, one might decide that path integrals only refer to one-dimensional systems, whereas functional integrals can be multi-dimensional - after all, only one-dimensional functions (functions only depending on time) can be interpreted as paths. If that distinction were to be made, the phrase "functional integral" would be more appropriate for this lecture, since path integrals in a one-dimensional target space formally represent the solution of ordinary partial differential equations, and as such they hardly present any formal difficulty. 1] In physics, our "integration variables" are often functions defined in a multi-dimensional base space, and this is where problems of a fundamental nature arise. We wish to integrate over entire sets of functions of several variables, not just "paths", which are functions of time only.

Functional integrals form the back bone of Quantum Field Theory, which is a widely applied approach in theoretical physics. In condensed matter physics both quantum mechanical and classical statistical models can be addressed using functional integrals, and in elementary particle physics the functional integral provided essential insight not only in understanding the gauge forces in what is now known as the Standard Model, but also in the many recent advances in Super String Theory and its successor, $M$-theory. All these successes made most theoreticians believe that, if there are any mathematical difficulties in defining and justifying the use of functional integrals, then that is something only for philosophers or mathematicians to worry about; physicists know what they are doing.

Extensive experimentation, both in condensed matter physics and in elementary particle physics appears to vindicate this attitude, but it does not justify blindness for the various complications that may arise. Indeed, as we shall argue, there is little experimental support for the use of functional integrals in dimensions greater than four, and the exact definitions will be extremely complex. Consequences of this situation for model building are often underestimated.

In Chapter 2, we address the important question, what is a functional integral? In general, an integral is defined to be the limit of an infinite sum; in turn, a functional integral is the limit of an infinite number of integrations. How do we know whether such a limit makes sense? More often than not, the formal definition does not address this limiting procedure properly.

In Chapter 3, it is shown that, apparently, the formal definition makes a lot of sense when the integrand is nearly Gaussian, while the non-Gaussian part is treated perturbatively. The difficulty here is well-known: infinities arise that have to be 'renormalized'. Often, there are severe prices to be paid for that.

For some special cases, renormalization works. In particular, if a theory turns out to be 'asymptotically free'. In Chapter 1 , we show that then, under some quite plausible assumptions, the functional integral allows for a nonperturbative definition. This, however, would limit us to theories where the dimensionality of target space is at most four. In higher dimensions, one has to make much more drastic - and less reliable - assumptions concerning the existence of equivalence classes and the absence of a mass gap at the lattice scale, which would be necessary for considering the transition towards the continuum limit.

Subsequently, two separate issues are briefly discussed: the duality transformation, popular nowadays in string theories (Chapter 5), and the Wick rotation (Chapter 6), which requires some detailed thoughts in the case of quantum gravity. Finally, some conclusions (Chapter 7 ). 


\section{Defining Functional Integrals.}

In principle, it may appear to be straightforward to provide a rigorous definition of what functional integrals are supposed to mean. Consider a set of functions $A_{i}(x)$, where $i$ is some discrete index that may take $N$ distinct values, and $x$ is an element of some multi-dimensional, continuous space-time, such as Minkowski space. Next, consider an integrand of the form

$$
e^{\int \mathrm{i} \mathcal{L}(x) \mathrm{d}^{n} x}
$$

where $n$ is the dimensionality of space-time, and the Lagrange density $\mathcal{L}(x)$ is generally of the form?

$$
\mathcal{L}(x)=-\frac{1}{2}\left(\partial_{\mu} A_{i}(x)\right)^{2}-\frac{1}{2} m_{(i)}^{2} A_{i}^{2}-\frac{1}{3 !} g_{i j k} A_{i} A_{j} A_{k}-\frac{1}{4 !} \lambda_{i j k \ell} A_{i} A_{j} A_{k} A_{\ell},
$$

where $g_{i j k}$ and $\lambda_{i j k \ell}$ are the coupling coefficients of the 'theory', and $m_{(i)}$ are possible mass terms (here already diagonalized). In gauge theories, $g_{i j k}$ may contain one space-derivative with respect to $x$.

We wish to integrate this integrand over the entire space of functions $A_{i}(x)$, but what does this mean? Ordinary integrals of functions $F(A)$ of a single variable $A$ can be defined very rigorously [2]: one takes the sum of the function values over a discrete and finite set of points in $A$ space while multiplying the integrand with the separation distance $\Delta A \stackrel{\text { def }}{=} A-A^{\prime}$ of neighboring points $A$ and $A^{\prime}$. The limit

$$
\lim _{\Delta A \downarrow 0} \sum_{A} \Delta A F(A)
$$

is defined to be the Lebesgue-integral $\int \mathrm{d} A F(A)$. If $A$ has an index $i=1, \cdots, N$, we simply repeat the procedure $N$ times, so that we obtain the $N$-dimensional integral, $\int \mathrm{d}^{N} \vec{A} F(\vec{A})$. But what do we have to do if $A$ depends on a continuous variable $x$ ? This $x$ takes an infinite number of values. What does it mean to repeat the procedure $\aleph_{1}$ times?

At first sight, the remedy appears to be a straightforward one: just introduce a grid in $x$ space as well. Once $x$-space has been replaced by a grid with a finite number of points in it, our "functional" integral has been reduced to an integral in a space with a finite number of dimensions, and the Riemann-Lebesgue definition of the integral is applicable. This time, however, it is far from straightforward to justify even the speculation that the limit for infinitely dense grids in $x$ space makes any sense at all.

There is one circumstance where a reasonable procedure seems to be possible. This is the case mentioned in the introduction, where $x$ is one-dimensional (typically a time coordinate). In this case, we can order the lattice points, and first consider the integration over all lattice points left of some value $t$ in $x$ space, while keeping $A(t)=A$ fixed. Assume this intermediate result to be some function $\psi(A, t)$. One then finds [1] that $\psi(A, t)$ obeys a partial differential equation, in fact the Schrödinger equation, where the Hamiltonian $H$ is the one associated to the Lagrangian (2.2).

Note, however, that this result is not obtained unless we define the measure of the integral with sufficient care while taking the limit where the grid in $x$-space is made infinitely dense. [3]

\footnotetext{
${ }^{2}$ For the time being, we ignore fermionic degrees of freedom; complications that are special to fermions are important, but their role in the issues raised here is of a purely technical rather than fundamental nature.
} 
The measure required is what became known as the Wiener measure. Note also that $H$ is expected to be the quantum Hamiltonian, and that its relation to the classical Hamiltonian generated by $\mathcal{L}$ might not be unambiguous due to ordering problems. An example in case is the path integral for a charged quantum particle in a magnetic field. Here, only a grid in one time variable is needed, but it must be set up in a way that is invariant under reversal of time, otherwise the resulting Hamiltonian may come out to be non Hermitean due to unmatched commutators. Thus, even the one-dimensional path integral contains some pit falls.

In general, however, the difficulties for the one-dimensional case can be kept well under control, and the theory of partial differential equations allows for a sufficiently rigorous treatment.

What, however, becomes of the Wiener measure when also $x$ is multi-dimensional? Here, one enters the subject of Quantum Field Theory. What is needed is a Wiener measure for the multi-dimensional case. This time, we cannot use the theory of partial differential equations in finite-dimensional spaces, but, at first sight, it appears that the generalization to a multidimensional Wiener measure is again straightforward. All we need to do is study very dense grids in $x$ space. Take a magnifying glass and study the 'theory' at a very tiny distance scale. In the one-dimensional case, this tiny distance scale was represented by the tiny time step $\Delta t$, turning into the infinitesimal quantity $\partial t$ in the partial differential equation $\partial \psi / \partial t=-i \hat{H} \psi$. Only if, in the multi-dimensional case, the small-distance limit would be similarly well-behaved, would we be able to define the functional integral there. In practice, one studies the theory on a grid with lattice length $a$, and studies the limit $a \downarrow 0$.

Let us focus on the limiting situation, confining ourselves only to a tiny region $R$ in spacetime. If $R$ is small enough then, inside $R$, our theory should be entirely featureless, or at least we should have the entire theory completely under control, at all scales, inside the region $R$. It is only at scales much larger than $R$, where we expect our theory to exhibit interesting physical features.

It is difficult to imagine that this could mean anything else than complete scale invariance inside the tiny region $R$, which in practice also implies conformal invariance. What we are talking about is what in the one-dimensional theory would simply be the domain where the solution to the partial differential equation behaves linearly in the time interval $\Delta t$. We postulate the existence of small enough regions $R$ in spacetime where such triviality occurs. Thus, the couplings $g_{i j k}$ and $\lambda_{i j k \ell}$ should approach fixed points in the ultra-violet region. In principle, there exists also the possibility of periodic behavior of these coupling strengths in their dependence of the logarithm of the scale of $R$. To be precise then, we define the 'bare' couplings to depend on the grid size $a$ in such a way that they either approach constant values or values periodic in $\log (a)$. Tiny deviations from the limiting values at small but finite $a$ should then lead to physically interesting structure in the physical scale.

It then remains to be proven that the scaling behavior of these extremely complicated integrals is as desired, and, as we shall see, this requirement will present us with rather fundamental problems, except when the number of dimensions is four or less.

As we shall argue later, not only is the periodic scaling behavior a problematic option, so that we should really demand the approach of a single fixed point, we shall even demand this fixed point either to be at the origin: $g_{i j k}$ and $\lambda_{i j k \ell} \rightarrow 0$, or that the fixed point values be very small. 


\section{Perturbation Expansion.}

The case that we presently really do have under control is when all couplings are sufficiently tiny to warrant a perturbative approach. In the absence of all couplings, the integral (2.1) is exactly Gaussian, and its value is easy to compute exactly. In the case when the Lagrangian $\mathcal{L}$ does contain non-quadratic contributions, we extract a quadratic part and expand the exponent of the remainder as a perturbative expansion in terms of (powers of) $g_{i j k}$ and $\lambda_{i j k \ell}$. The contributions of these terms in the perturbation series to an amplitude are usually represented as Feynman diagrams.

Computation of the contribution of a given Feynman diagram requires the evaluation of integrals in momentum space that often appear to be divergent, and these divergences appear to become more serious as we move to higher dimensions. The most harmful of these divergences are the ultra-violet ones, which occur at large values of the intermediate momenta $k_{\mu}$, and thus they refer to the apparent unboundedness of the effects coming from very tiny distance scales. This really means that the small-distance limit referred to in the previous Section is not at all as straightforward as one might think.

The situation is improved considerably by renormalization. In principle, what renormalization means is that the limit where the grid line distance $a$ tends to zero has to be taken in such a way that the values of most, if not all, "physical" parameters of the theory, such as the coupling strengths $g_{i j k}$ and $\lambda_{i j k \ell}$, as well as possible mass terms $m_{(i)}$ in Eq. (2.2), are carefully tuned to run either to infinity or to zero during the limiting process. Also, physical operators such as the field operators $A_{i}(x)$, have to be renormalized. If no further adjustments are needed for the limit to exist, the theory is called 'renormalizable'. In the '60s and early '70s, elementary particle physicists worked out how renormalization works in combination with the perturbation expansion, and the required limiting procedure was identified for the complete set of models that can be called perturbatively renormalizable. [⿴囗口

The conditions for a field theory to be perturbatively renormalizable can be summarized very easily. Since we usually put $c=\hbar=1$, there is only one dimensionful unit required to gauge the physical parameters, Usually, we take this to be a length scale, say a $\mathrm{cm}$ or a Fermi. We now must require that all physical parameters in terms of which we need to do a perturbation expansion, have a dimension of length raised to some power that is either negative or zero. This can easily be understood: this dimensionality assures that, at extremely tiny distance scales, the effects of these coefficients can be ignored, or they stay sufficiently small (in case the dimension were zero); thus, the theory tends to a free theory in the far ultra-violet, and the limiting process described earlier can be carried out successfully.

The scaling dimension for the physical parameters of a theory can be found as follows. In $n$ space-time dimensions, the Lagrange density function $\mathcal{L}$ of Eqs, (2.1) and (2.2) has the dimensionality of $[\text { length }]^{-n}$, which will be indicated as "length dimension $-n$ ", or "mass dimension $n$ ". From the first, kinetic term in Eq. (2.2), which carries no physical constants, it follows that the fields $A_{i}$ have mass dimension $(n-2) / 2$, and subsequently that the cubic couplings $g_{i j k}$ (if not associated with space-time derivatives) will have mass dimension

$$
n-\frac{3}{2}(n-2)=3-n / 2,
$$

and the quartic couplings have mass dimension

$$
n-\frac{4}{2}(n-2)=4-n .
$$


In four space-time dimensions $(n=4)$, these are the only couplings with non-negative mass dimension. All renormalizable theories in four space-time dimensions have at most quartic polynomials in their Lagrangians!

The only reason why it took us several decades to work out the technical details of the procedure needed to get into grips with the small-distance structure of models for elementary particles, and to renormalize them properly, was an apparent clash between Lorentz invariance and local gauge-invariance. A simple grid in space-time would suspend Lorentz invariance, so that horrendously complicated intermediate results would obscure the proofs. Consequently, a battery of different, more symmetric cut-off procedures were invented, each having more elegant symmetry, but most of them either violated local gauge invariance, or unitarity, while both local gauge invariance and unitarity are necessary to ensure not only that our theories are stable, but also that we can limit ourselves to interactions with the right dimension. Thus, we had to find out how to link various different kinds of cut-off (or 'regularization') procedures.

One of the most universally applicable cut-off procedures turns out to be 'dimensional renormalization'. [5] If we take the number of space-time dimensions slightly away from the

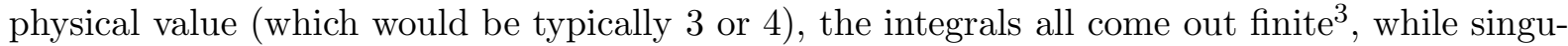
larities that develop when the physical number of dimensions is approached can be renormalized separately. Using this dimensional renormalization, one sees that, naturally, coupling parameters that are dimensionless in the physical space-time dimensions, nevertheless need to be renormalized, and that the 'bare' constants to be used at the cut-off scale tend to depend logarithmically on this scale.

\section{The non-perturbative definition.}

Although, by construction, the renormalization procedure was always linked to the perturbation expansion, we now see that its importance transcends perturbation theory. The point is that the ultraviolet limit of the theory should be required to be virtually non-interacting, which means that, just because perturbation expansion can be applied there, the smooth limit condition of the previous Section should be fulfilled. If the perturbative renormalizability condition is not met, we know for sure that the effective interaction strengths at short distances will run out of the domain where they can be handled perturbatively.

The theory is renormalizable in the usual sense, if all physical parameters have negative or zero length dimension, but this still leaves two distinct possibilities at the extremely tiny distance scales. The dimensionless bare coupling parameters depend on the cut-off scale logarithmically. In the far-ultraviolet, these so-called 'running coupling parameters' may either run away from the perturbative domain, or they all run to zero.

If the parameters run away from the perturbative domain, we formally have the same situation as in non-renormalizable theories. In practice, however, these models are still superior to non-renormalizable theories, because the logarithmic scale dependence is extremely slow as long as the couplings are weak. This allows one to perform extremely accurate calculations even if the mathematical basis is imperfect. One cannot make the space-time grid infinitely dense, but clashes only occur at scales of the order of $\exp (-C / \lambda)$, where $\lambda$ is a typical coupling parameter, and if $\lambda$ is sufficiently small, this is completely negligible. A typical example of a theory where

\footnotetext{
${ }^{3}$ More precisely: the finite and infinite parts of the integrals can be separated from one another unambiguously, and the infinite parts cancel completely upon renormalization.
} 
accurate calculations can be done even if the mathematical basis suffers from this difficulty is Quantum Electrodynamics. Most researchers agree that the likely explanation for its successes is that QED is an 'effective' theory; particles and forces that radically modify the physics at ultrashort distance scales have simply been ignored.

\subsection{The asymptotically free case.}

Theories where all couplings run to zero in the ultraviolet ("asymptotic freedom") are in a superior shape. [6] [7] [8] Here, we believe that the short-distance behavior is completely under control. Formally, the short-distance domain is described by perturbation theory. If $\mu$ is the mass scale at tiny distances (the distance scale $\ell$ simply being defined as $1 / \mu$ ), then the running couplings $\lambda(\mu)$, or, in gauge theories, $g^{2}(\mu)$, obey equations such as

$$
\mu \frac{\mathrm{d}}{\mathrm{d} \mu} \lambda(\mu)=-\left|\beta_{2}\right| \lambda^{2}+\beta_{3} \lambda^{3}+\delta \beta(\lambda)
$$

where $\delta \beta(\lambda)$ stands for higher order terms in this equation. We see that solutions look as follows:

$$
\frac{1}{\lambda(\mu)}=\left|\beta_{2}\right| \log \mu+\frac{\beta_{3}}{\left|\beta_{2}\right|} \log \lambda(\mu)+\mathcal{O}\left(\int_{0}^{\lambda(\mu)} \frac{\mathrm{d} \lambda \delta \beta(\lambda)}{\left|\beta_{2}\right| \lambda^{4}}\right)+\frac{1}{\lambda_{0}}
$$

where $\lambda(\mu)$ at the r.h.s. must be eliminated recursively, which is unambiguous as long as $\lambda$ is small enough. $\lambda_{0}$ is some fixed integration constant. If here, $\delta \beta(\lambda)$ is interpreted as some higher order disturbance, then we observe that all higher order effects that vanish as $\lambda^{4}$ or faster, for small $\lambda$, will not seriously affect the limiting expressions. As soon as the coefficients $\beta_{2}$ and $\beta_{3}$ are known, Eq. (4.2) can be used to define the running coupling parameter $\lambda(\mu)$, by fixing the integration constant $\lambda_{0}$. To give this definition, $\beta_{3}$ was explicitly needed, because of the divergence of $\log \lambda$, whereas the details of the quartic corrections $\delta \beta(\lambda)$ are not needed to be known explicitly; they are to be absorbed in the definition of $\lambda_{0}$.

It is tempting to assume that all higher order corrections to the amplitudes, beyond those of the irreducible two-loop diagrams, can therefore be absorbed into tiny redefinitions of the coupling constant $\lambda_{0}$, but this remains to be proven. To illustrate how difficult this problem is: instanton effects are known to be associated with a new, free constant of Nature, the instanton angle $\theta$, whereas their amplitudes are proportional to $\exp \left(-C^{\text {nst }} / \lambda\right)$, which tends to zero much faster than $\lambda^{4}$; we could have missed these effects if the above definition were trusted blindly. Attempting to use our definition of the functional integral in terms of the "physical constant" $\lambda_{0}$ yields expressions of which the convergence could still not be proven. This is a fundamental problem for theories such as QCD, in spite of its phenomenal success at describing the strong interactions among hadrons.

In spite of the absence of rigorous mathematical proofs, there appears to be no serious difficulty in practice in the use of theories such as QCD. Experimentally, the agreements are impressive. We strongly suspect therefore that the definition of what a functional integral is, in the case of an asymptotically free quantum field theory, is an acceptable one.

A powerful argument in favor of the suspicion that the mathematical definition of asymptotically free theories, starting from a running coupling strength $\lambda(\mu)$, is unambiguous, is the following: Imagine that two different theories existed that both were described by the same 
scaling limit. It should be possible to trace this difference to differences in the effective interactions at tiny distance scales. Could we write a model describing this effective interaction $\Delta \mathcal{L}(x)$ at small distances? Unitarity and causality would demand that $\Delta \mathcal{L}$ has the form of a strictly local (effective) Lagrangian. It should be different from the defining Lagrangian of the theory. If the defining Lagrangian contains all possible terms with the right symmetry and dimensionality, the only possibilities left for $\Delta \mathcal{L}$ is some effective Lagrangian of a higher dimensionality, such as one containing higher derivatives or higher powers of the fields. Interactions of this sort are usually called marginal terms in the interaction Lagrangian. Terms with lower dimensionality do not exist. Since marginal terms, which have higher dimensionality than the canonical ones, would diverge at tiny distances, while the amplitudes there had been postulated to have the desired scaling behavior, we can exclude the presence of such exotic effective interactions, ergo, the theory must be a unique one.

\subsection{Theories with strong interactions in the UV limit.}

According to perturbation theory, models that are not asymptotically free can still be described by coupling parameters that run as a function of the scale $\mu$ :

$$
\mu \frac{\mathrm{d}}{\mathrm{d} \mu} \lambda(\mu)=\beta(\lambda(\mu))
$$

where the function $\beta(\lambda)$ can be computed perturbatively. If $\beta$ is negative, the theory is asymptotically free. Let us now assume $\beta(\lambda)$ to be positive. After having computed the first few terms of the expansion $\beta(\lambda)$ for small $\lambda$, one may find that the function appears to sport a zero at some finite value of $\lambda$ :

$$
\beta\left(\lambda_{0}\right)=0 .
$$

This zero then is an attractive one in the UV direction. If the coupling has the value $\lambda_{0}$, the theory is scale-invariant (which usually implies also conformal invariance). As we desire to give a rigorous definition of the theory, we put it on a lattice, as before, and give the 'bare' coupling parameter the value $\lambda_{0}$ (with possibly a small correction). It is tempting then to assume that, indeed, the amplitudes will reproduce the scale invariance. But now, there are several problems.

First, just because the coupling is never very small, the artifacts due to the finite lattice size are complicated to compute, and they will be non-negligible. They may invalidate the argument that the theory scales, so that the limit where the lattice length $a$ tends to zero is not well under control.

Secondly, if the coupling is strong, its actual value depends strongly on many details in its definition. In perturbation theory, we see this as a dependence on the subtraction procedure, at every order of the calculations; in the lattice theory we see this as a dependence on details of the definition of the lattice.

However, theories that have a lattice with strong interactions in the far ultraviolet domain (that is, a very dense lattice), and which do tend to become scale-invariant in the far infrared (i.e. at large distances) do exist. We know that perturbative schemes exist where the coupling constant $\lambda_{0}$ can be made small but non-zero. If such a theory is put on a lattice, then we have an example of a non-asymptotically free theory that is presumably well-defined.

Can such non-asymptotically free theories be unique? Again, we can attempt to argue that any non-uniqueness would be described by effective interactions with anomalous dimensions. 
This time, however, we cannot use perturbation theory at the ultraviolet as a guide. If the theory is 'nearly' asymptotically free, that is, $\lambda_{0}$ is small, the argument appears to be as reliable as in the asymptotically free case, but if $\lambda_{0}$ is large, we have no clue.

Even worse will the situation be if there is no fixed point at all, but instead a limit cycle. This may happen in the case of multiple coupling parameters, which in the ultraviolet domain tend to a periodic solution of Eq. (4.3). Here, much less is known. If such theories do exist, and if they can be demonstrated to be unique, then we should be able to list them as universality classes, much like what is done in statistical mechanics in three space dimensions. Again, we must assume that marginal terms can be excluded.

An important case is quantum field theory in more than four space-time dimensions. Here, we see that all quartic terms in the lagrangian have negative mass dimension (see Eq. (3.2)). We must have quartic terms (if not higher) if we want the Hamiltonian of the theory to be bounded from below, so that the system is stable. This implies that the function $\beta(\lambda)$ starts off with a term $(n-4) \lambda$, which is positive. To develop a zero in this function, we need the higher order terms and hence the coupling can never be small. This also holds if we want a limit cycle. Thus, in more than four space-time dimensions it is inevitable to have strong coupling at the cut-off point. One may even question whether a zero in $\beta(\lambda)$ can ever occur. In any case, the UV limit cannot be treated using perturbation theory. It can only be treated by speculation. The only alternative would be a numerical experiment using computers, but now the problem is that, precisely in a large number of space-time dimensions, numerical algorithms tend to become prohibitively slow.

A superior approach to the questions at hand is to start from some generic lattice theory that exhibits the required symmetry properties of the continuum theory one wants to study. Then we should ask: is there any set of values for the various coupling parameters such that there is a non-trivial far infrared region, where the theory becomes scale-invariant yet non-trivial? If such a set is found, one can subsequently consider a slight deviation from these values, which will break the infrared scale invariance, thus producing effective mass terms, and with those, more non-trivial structure. Looking upon our problem this way, it is evident that success depends on the existence of such a set of coupling parameters. It definitely does not exist within the perturbative domain, if $n>4$. One might conclude for these reasons that the existence of any consistent quantum field theory at $n>4$ should be dubious. This should even include supersymmetric theories. Supersymmetry is difficult - if not impossible - to reproduce on a lattice.

But we could also speculate on the existence of fine-tuned theories that do survive in some non-trivial manner. These will be theories with strong interactions throughout, often with scaleinvariance and possibly with other special symmetries such as supersymmetry. They may form universality classes. In the next Section, we speculate that the number of distinct universality classes could be smaller than what is suggested by writing down Lagrangians.

\section{5. duality}

Again, first consider theories with a rigorous lattice cut-off. In such systems, different systems may exhibit dual relationships. The simplest example of such a relationship is the 2-dimensional Ising Model, where the coupling parameter $\beta$ can be given any value from $-\infty$ to $+\infty$. By rearrangement of the primary degrees of freedom, one finds that all properties of the model wih 
given $\beta$ can be mapped onto the features of the same model, living on the dual of the original lattice , at the coupling $\beta^{*}$, where $\beta^{*}$ is related to $\beta$ as 10

$$
e^{2 \beta^{*}}=\operatorname{coth} \beta \text {. }
$$

The relation is dual in the sense that, when applied twice, it returns to the original model.

Relations of this sort are more general. In three dimensional lattice theories, one finds a similar dual relation linking two different models: the Ising Model is dually related to the $\mathbb{Z}_{2}$ theory on the dual lattice. In four dimensions, the $\mathbb{Z}_{2}$ theory is self-dual.

Duality on the lattice is not restricted to $\mathbb{Z}_{2}$ theories, but, for its rigorous definition, it does require an Abelian structure. In fact, Eq. (5.1) is the simplest example of a Fourier transform in parameter space. If the link variable can be written as a commuting quantity $\sigma$, and the action is written as

$$
\sum_{\text {links } \ell} \beta\left(\sigma_{\ell}\right)
$$

then the dual theory obtains the same action, but with $\beta^{*}\left(\sigma_{\ell}^{\prime}\right)$, defined by

$$
Z e^{\beta^{*}\left(\sigma^{\prime}\right)}=\sum_{\sigma} e^{i \sigma \sigma^{\prime}} e^{\beta(\sigma)}
$$

where $Z$ is a normalization factor. The duality transformation (5.3) can be generalized even more by having a higher-dimensional $\sigma$ field.

These observations would allow us to perform dual transformations on a variety of theories, which however all have to be Abelian. The Fourier transformation in Eq. (5.3) is a linear transformation, and there seems to exist no direct generalization towards non-Abelian systems. Thus, in four space-time dimensions, only Abelian gauge theories can be dually transformed to other Abelian gauge theories. What we can do for non-Abelian theories is first integrate away their non-Abelian parts. This is indeed exactly what is done in the procedure called Abelian projection in QCD. 9] The Abelian, or Cartesian subgroup of the gauge group can be seen to correspond to an ordinary Abelian gauge theory, to which the non-Abelian sector adds not only electrically charged objects, but also magnetic monopoles. The dual transformation then interchanges the monopoles and the electric charges.

Unfortunately, it appears to be impossible to carry out such a transformation procedure exactly. It is therefore quite remarkable that nevertheless dual transformations among supersymmetric gauge theories appear to be possible, provided that one restricts oneself to the far infrared domain. a possible explanation for the remarkable facts that were discovered is that the dual transformations only hold for the universality classes, not for the individual theories with any finite cut-off.

\section{The Wick Rotation.}

An important calculational tool in many quantum field theories is the Wick rotation. First, one notices that, in Feynman diagrams, rotational symmetry can be exploited more fully by substituting

$$
k_{0} \quad \rightarrow \quad k_{4}=\mathrm{i} k_{0} .
$$

\footnotetext{
${ }^{4}$ The dual lattice is obtained from the original lattice by interchanging the plaquettes with the lattice sites.
} 
Here, this is not more than a simple contour rotation in the complex plane of the integration variable $k_{0}$. In coordinate space, the equivalent rotation is

$$
t \rightarrow \tau=\mathrm{i} t .
$$

The functional integral expression, for instance in a gauge theory, is then replaced as follows:

$$
\int \mathcal{D} A e^{-\mathrm{i} \int \frac{1}{4} F_{\mu \nu} F_{\mu \nu} \mathrm{d}^{n-1} \vec{x} \mathrm{~d} t} \rightarrow \int \mathcal{D} A e^{-\frac{1}{4} F_{\mu \nu} F_{\mu \nu} \mathrm{d}^{n-1} \vec{x} \mathrm{~d} \tau} .
$$

In all conventional quantum field theories, the complex integrand turns into a Gaussian integrand, and, being the exponential of a negative quantity, the integrals converge optimally.

In the case of the gravitational field, however, things work out differently. At first sight, one is tempted to proceed in exactly the same way. The substitution $t \rightarrow \tau=\mathrm{i} t$ can be performed in the Einstein-Hilbert action. In the functional integral for what should become a quantum theory of gravity, one [erforms the switch

$$
\int \mathcal{D} g_{\mu \nu} e^{\mathrm{i} \int \sqrt{-g} R \mathrm{~d}^{n-1} \vec{x} \mathrm{~d} t} \rightarrow \int \mathcal{D} g_{\mu \nu} e^{\int \sqrt{g} R \mathrm{~d}^{n-1} \vec{x} \mathrm{~d} \tau}
$$

The difficulty here is that the Einstein-Hilbert action, $\sqrt{g} R$, is not at all bounded from below, not even after rotating all i's away. Consequently, the resulting integration expression, Eq. (6.4), makes no sense at all.

Elaborate proposals have been formulated to turn this meaningless expression into something one can calculate; the problem is sometimes believed to cure itself, somehow. This, however, is not the correct answer. The correct answer is found by returning to the roots of the procedure: rotating integration contours in the complex plane. In ordinary perturbation theory, one sees most easily what happens. The rule here is: if you have an integral that converges because the integrand becomes rapidly oscillating at infinity, you can obtain an equivalent expression that converges faster by rotating the integration contour in the complex plane. The rotation must be performed in such a way that, while rotating the contour over a variable angle $\varphi$, the integrand converges at infinity. In practice, this means that integration variables may be chosen to rotate in the complex plane, but this must always be done in such a way that the integral becomes an exponentially convergent one.

Let us take four dimensional space-time, to be explicit. If we rotate the metric field variables,

$$
\int \mathcal{D} g_{\mu \nu}=\prod_{x} \int_{C 1} \mathrm{~d} g_{00}(x) \int_{C 2} \mathrm{~d} g_{01}(x) \cdots \int_{C 10} \mathrm{~d} g_{33}(x)
$$

we must ensure that the resulting integrals converge. Now, because of local gauge invariance, we must impose a gauge condition, and add the usual ghost term. Just as in Maxwell theory, after fixing the gauge, not all surviving degrees of freedom are truly dynamical. Some of them act as Lagrange multipliers. In Maxwell theory, of the four vector components of the vector potential field $A_{\mu}$, one disappears as a consequence of the gauge condition, and an other one turns into a Lagrange multiplier to produce the Coulomb potential. Two physical photonic degrees of freedom survive. In gravity, there are 10 components of $g_{\mu \nu}$, and 4 gauge conditions (fixing the 4 coordinates). Of the 6 surviving fields, 4 act as Lagrange multipilers, so that two graviton degrees of freedom remain.

In perturbation theory, one sees most clearly that some of these Lagrange multipliers should not rotate so that the metric would become nonnegative. Although the details depend on 
how the gauge was fixed, it appears in general not to be possible to avoid contours to rotate incorrectly, unless we keep the metric complex. This is easy to understand: this happens because the Einstein-Hilbert action is unbounded!

In many gauge choices, one finds that only the conformal factor in the metric needs to be complex. Thus, we write

$$
\int \mathcal{D} g_{\mu \nu} \rightarrow \prod_{x} \oint \mathrm{d} \Omega \prod_{\mu \nu} \mathrm{d} \hat{g}_{\mu \nu}
$$

where

$$
g_{\mu \nu} \equiv \Omega \hat{g}_{\mu \nu}, \quad \text { and } \quad R\left(\hat{g}_{\mu \nu}\right) \equiv 0 .
$$

\section{Conclusion.}

The possibility to define functional integrals in more than one space-time dimensions depends on the existence of universality classes. In less than four dimensions, these classes are relatively easy to define, since the far ultraviolet (i.e. small-distance) domain of the theory is controlled by perturbation theory, which we know how to handle. In four dimensions, this is also the case if we have asymptotic freedom, or possibly if the coupling strength tends to a zero of the $\beta$ function where it is itself small, so that one may still rely on perturbation theory to find a useful theory.

However, in particle theory, and notably in string theory and in supergravity, one often speculates on theories in much more than four space-time dimensions. The possibility to define what a functional integral is, depends on features one can only speculate about.

What we know for certain about our physical world is that functional integrals successfully describe statistical systems in three space-dimensions and elementary particles in four space-time dimensions.

If there exist more dimensions describing physics at ultra-tiny scales, then well-defined functional integrals in more than four dimensions would be needed there. Such theories can only stretch over a large domain of scales if, at the largest distance scales, the effective interactions are either extremely weak (since the only allowed effective interactions are marginal ones), or extremely fine-tuned: the physical interaction parameters are very strong, and they are tuned in such a way that the theory scales. Models based on continuum physics but nevertheless exhibiting interesting non-trivial interactions, in more than four dimensions, are therefore physically unrealistic, and this may explain why, as yet, no experimental evidence has been found in favor of Kaluza-Klein theories for elementary particles.

The Wick rotation in quantum gravity is less enigmatic than what is often claimed, but the real physical significance of quantum wave functions on complex conformal factors may have to be investigated further.

\section{References}

[1] R.P. Feynman, Rev. Mod. Phys. 20 (1948) 367.

[2] C.D. Aliprantis and O. Burkinshaw, Principles of Real Analysis, 1981 Elsevier N.H. Inc., London, ISBN 0713134348. 
[3] C. Garrod, Statistical Mechanics and Thermodynamics, Oxford Univ. Press 1995, ISBN 0 19 508523-X / 509775-O.

[4] G. 't Hooft and M. Veltman, "DIAGRAMMAR", CERN Report 73/9 (1973), reprinted in "Particle Interactions at Very High Energies, Nato Adv. Study Inst. Series, Sect. B, vol. 4b, p. 177.

[5] G. 't Hooft and M. Veltman, Nucl. Phys. B44 (1972) 189.

[6] H.D. Politzer, Phys. Rev. Lett. 30 (1973) 1346; id., Phys. Repts. 14c (1974) 129.

[7] D.J. Gross and F. Wilczek, Phys. Rev. Lett. 30 (1973) 1343.

[8] G. 't Hooft, Nucl. Phys. B61 (1973) 455.

[9] G. 't Hooft, Nucl. Phys. B190[FS3] (1981) 455.

[10] H.A. Kramers and G.H. Wannier, Phys. Rev. 60 (1941) 252. 